Published in final edited form as:

Cancer Epidemiol Biomarkers Prev. 2008 August ; 17(8): 2123-2127. doi:

10.1158/1055-9965.EPI-07-2735.

\title{
Variants of DNA Repair Genes and the Risk of Biliary Tract Cancers and Stones: A population-based study in China
}

\author{
Mingdong Zhang ${ }^{1}$, Wen-Yi Huang ${ }^{2}$, Gabriella Andreotti ${ }^{2}$, Yu-Tang Gao ${ }^{3}$, Asif Rashid ${ }^{4}$, \\ Jinbo Chen ${ }^{5}$, Lori C. Sakoda 6 , Ming-Chang Shen ${ }^{7}$, Bing-Sheng Wang ${ }^{8}$, Stephen \\ Chanock $^{2,9,10}$, and Ann W. Hsing ${ }^{2}$ \\ ${ }^{1}$ Food and Drug Administration, Silver Spring, MD, USA \\ ${ }^{2}$ Division of Cancer Epidemiology and Genetics, National Cancer Institute, National Institutes of \\ Health, DHHS, Bethesda, MD, USA \\ ${ }^{3}$ Department of Epidemiology, Shanghai Cancer Institute, Shanghai, China \\ ${ }^{4}$ Department of Pathology, M.D. Anderson Cancer Center, Houston, TX, USA \\ ${ }^{5}$ Department of Epidemiology and Biostatistics, University of Pennsylvania, PA, USA \\ ${ }^{6}$ Department of Epidemiology, University of Washington, WA, USA \\ ${ }^{7}$ Shanghai Tumor Hospital, Fudan University, Shanghai, China \\ ${ }^{8}$ Zhongshan Hospital, Fudan University, Shanghai, China \\ ${ }^{9}$ Core Genotyping Facility, National Cancer Institute, National Institutes of Health, Gaithersburg, \\ MD, USA \\ ${ }^{10}$ Pediatric Oncology Branch, Center for Cancer Research, National Cancer Institute, National \\ Institutes of Health, Bethesda, Maryland, USA
}

\section{Abstract}

Biliary tract cancers, encompassing tumors of the gallbladder, extrahepatic ducts, and ampulla of Vater, are relatively rare tumors with a high fatality rate. Other than a close link with gallstones, the etiology of biliary tract cancers is poorly understood. We conducted a population-based casecontrol study in Shanghai, China, to examine whether genetic variants in several DNA repair genes are associated with biliary tract cancers or biliary stones. Genomic DNA from 411 patients with biliary tract cancers (237 gallbladder, 127 bile duct, and 47 ampulla of Vater), 891 patients with biliary stones, and 786 healthy subjects randomly selected from the Shanghai population, was genotyped for 6 single nucleotide polymorphisms (SNPs) in 4 DNA repair genes (MGMT, $R A D 23 B, C C N H$, and XRCC3). Of the 6 SNPs, only one (MGMT EX5-25C >T, rs12917) was associated with biliary tract cancer. Independent of gallstones, subjects carrying the CT genotype of the MGMT EX5-25C > T marker had a significantly reduced risk of gallbladder cancer (odds ratio $(\mathrm{OR})=0.63 ; 95 \%$ confidence interval $\left.(\mathrm{CI}): 0.41-0.97 ; P_{\text {trend }}=0.02\right)$ and non-significant reduced risks of bile duct $(\mathrm{OR}=0.61 ; 95 \% \mathrm{CI}$ : $0.35-1.06)$ and ampulla of Vater $(\mathrm{OR}=0.85 ; 95 \% \mathrm{CI}$ : $0.39-1.87)$ cancers. However, this marker was not associated with biliary stones, and the other markers examined were not significantly associated with either biliary tract cancers or stones. Findings from this population-based study in Shanghai suggest that MGMT gene variants may

Request for reprints: Ann W. Hsing, Ph.D., Division of Cancer Epidemiology and Genetics, National Cancer Institute, National Institutes of Health, 6120 Executive Blvd., EPS 7058, MSC 7324, Bethesda, MD 20892-7234. Phone: 301-496-1691; Fax: 301-402-0916. hsinga@mail.nih.gov. . 
alter susceptibility to biliary tract cancer, particularly gallbladder cancer. Confirmation in future studies, however, is required.

\section{Keywords}

Biliary tract cancers; gallbladder; extrahepatic bile duct; ampulla of Vater; DNA repair; polymorphism; China

\section{INTRODUCTION}

Biliary tract cancers, encompassing cancers of the gallbladder, extrahepatic bile ducts, and ampulla of Vater, are rare malignancies with a poor prognosis. Apart from gallstones, the risk factors for biliary tract cancers are not clearly defined. Since each of the three cancer subsites has distinct molecular changes as well as varying geographic and ethnic patterns, it has been suggested that there is a different etiology for each of the three subsites (1).

Disruptions in genomic stability and integrity, due to DNA damage, compromise the accuracy of DNA replication, resulting in gene rearrangements, translocations, amplifications, and deletions. These changes can contribute to cancer development $(2,3)$. DNA damage and mutations are induced by a variety of endogenous processes and of exogenous factors, including ultraviolet light, cigarette smoke, and dietary elements (4). Under normal circumstances, DNA damage and normal replication errors are corrected by DNA repair mechanisms.

To gain insight into the role of DNA repair in biliary tract cancer etiology, we investigated 6 variants in 4 DNA repair genes, including $\mathrm{O}^{6}$-methylguanine-DNA-methyltransferase $(M G M T)$, radiation gene $(R A D 23 B)$, cyclin $\mathrm{H}(C C N H)$, and the X-ray repair crosscomplementary group (XRCC3), and their associations with biliary tract cancer risk in a population-based case-control study in Shanghai, China.

\section{MATERIALS AND METHODS}

\section{Study Population}

The study protocol was approved by the Institutional Review Boards of the U.S. National Cancer Institute (NCI) and the Shanghai Cancer Institute (SCI). All participants provided written informed consent. Details of this population-based case-control study have been reported previously $(5,6,7,8)$. Briefly, cancer cases were permanent residents of urban Shanghai, 35-74 years of age, and newly diagnosed with biliary tract cancer between 1997 and 2001. Cancer cases were identified through a rapid reporting system established by the Shanghai Cancer Institute and 42 collaborating hospitals, which captured over $95 \%$ of the biliary cancer cases diagnosed in urban Shanghai during the study period. Biliary stone patients without a history of cancer were selected by frequency matching to cancer cases on age (5-year intervals), gender, and hospital. Population controls without a history of cancer were randomly selected from all permanent residents listed in the Shanghai Resident Registry and frequency-matched to cancer cases on age (5-year intervals) and gender. Of the eligible cancer cases and controls, $95 \%$ and $82 \%$ agreed to participate in the study, respectively.

For cancer cases, over $70 \%$ of subjects had positive pathology confirming their diagnosis. For each case with pathology materials, a second independent review and a consensus review were carried out to confirm the diagnosis. For each subject without pathological confirmation, medical records, surgical reports, and imaging data, including magnetic 
resonance imaging (MRI), endoscopic retrograde cholangiopancreatography (ERCP), and computed tomography (CT), were reviewed to confirm their diagnosis. Biliary stone cases were confirmed by review of abdominal ultrasound, ERCP films, medical records, and surgical records, or by pathologic material for those who underwent a cholecystectomy.

Gallstone status was assessed in nearly all biliary tract cancer cases and population controls. Gallstones were identified among cancer cases by self-reported history, surgical reports, or imaging results from MRI, ERCP, CT, or ultrasound. Among population controls, gallstones were identified by self-reported history or by abdominal ultrasound, among those who gave consent for the procedure ( $85 \%$ of all population controls).

\section{Interview}

In-person interviews were conducted with each participant by trained interviewers using a structured questionnaire to collect information on demographic characteristics, medical histories, and lifestyle factors, including diet and physical activity. Weight and height were measured at interview. Cases were interviewed within 3 weeks of diagnosis. All interviews were taped and verified for accuracy of interview protocol and coding. The response rate for interviews was over $95 \%$ among cases and $82 \%$ among controls. To assess the reproducibility of interview responses, $5 \%$ of the study subjects were randomly selected for a second interview three months after the initial interview. The concordance between the original and follow-up interviews was greater than $90 \%$.

\section{Genotyping}

Overnight fasting blood samples were collected from the participants who gave consent for blood collection (over $80 \%$ of the total group). For the current study, genomic DNA was available for the genotyping of 411 patients with biliary tract cancers (237 gallbladder, 127 bile duct, and 47 ampulla of Vater), 891 patients with biliary stones (670 gallstones and 221 bile duct stones), and 786 healthy subjects randomly selected from the Shanghai population. Genomic DNA was extracted from buffy coat at the NCI laboratory by the standard phenol chloroform method. Genotyping was performed at the NCI Core Genotyping Facility using the TaqMan assays (Applied Biosystems, Foster City, CA, http://snp500cancer.nci.nih.gov) (9). The following 6 single nucleotide polymorphisms (SNPs) in 4 genes in the DNA repair pathway were typed: $\mathrm{O}^{6}$-methylguanine-DNA-methyltransferase, MGMT EX5-25C $>\mathrm{T}$ (rs12917), the radiation gene, $R A D 23 B$ IVS5-15A $>\mathrm{G}$ (rs1805335), $R A D 23 B \mathrm{EX} 7+65 \mathrm{C}>\mathrm{T}$ (rs1805329), the cyclin $\mathrm{H}, C C N H \mathrm{EX} 7+49 \mathrm{~T}>\mathrm{C}$ (rs2266690), and the X-ray repair crosscomplementary group XRCC3 EX8-53C > T (rs861539). To evaluate the quality of genotyping, DNA aliquots were shipped to CGF with 80 internal blind duplicates from four individuals spaced at varying intervals between the study samples. The genotyping failure rate among all subjects was less than $2 \%$, and the concordance between quality control samples was over $99 \%$.

\section{Statistical Analysis}

Genotype frequencies for each polymorphism were examined among population controls for deviation from Hardy-Weinberg equilibrium (HWE), using the $\chi^{2}$ test. Unconditional logistic regression was used to calculate odds ratios (ORs) and 95\% confidence intervals ( $95 \%$ CIs) to assess the relationship of each polymorphism with biliary tract cancer and stone risk at each anatomic subsite, adjusting for age and gender. For biliary tract cancer risk, additional logistic regression models were run with further adjustment for biliary stone status, to evaluate potential confounding by this factor. Estimates were calculated for the heterozygous, homozygous, and their combined genotypes, with the homozygous genotype of the more frequent allele as the referent category for each marker. Bile duct and ampulla of Vater cancer cases were compared with all population controls. Gallbladder cancer cases 
were compared with controls without a history of cholecystectomy, and gallstone and bile duct stone cases were compared with controls without biliary stones.

Because preserved foods contain nitrosamines whose DNA damaging effects are mitigated by DNA repair mechanisms, we examined the joint effects of the DNA repair variants and the consumption of preserved foods on biliary tract cancer and stone risk. Preserved food consumption (g/day) was dichotomized into $<14.4 \mathrm{~g} /$ day and $\geq 14.4 \mathrm{~g} /$ day, based on the median consumption among the control subjects. We also examined whether the effect genetic variants on biliary tract cancer or stone risk was modified by other potential risk or protective factors, such as regular cigarette smoking (no, yes), BMI $\left(<23, \geq 23 \mathrm{~kg} / \mathrm{m}^{2}\right.$ ), intake of red meat ( $<30.1 \mathrm{~g} /$ day, $\geq 30.1 \mathrm{~g} /$ day), allium vegetables ( $<9.7 \mathrm{~g} /$ day, $\geq 9.7 \mathrm{~g} /$ day $)$, all vegetables ( $<720.7 \mathrm{~g} / \mathrm{day}, \geq 720.7 \mathrm{~g} / \mathrm{day})$, all fruits ( $<653.6 \mathrm{~g} / \mathrm{day}, \geq 653.6 \mathrm{~g} / \mathrm{day})$, and gallstones for cancer risk only (no, yes). To maximize statistical power in exploring potential interactions, we assumed the dominant model, with heterozygote and homozygous variant carriers combined, and selected characteristics were categorized dichotomously based on the median among the controls. Multiple interactions were assessed, using a genotype-exposure cross-product term in the logistic regression models. All analyses were performed using SAS version 8.0 (Cary, NC). All reported p-values are two-sided.

\section{RESULTS}

Selected characteristics of the study subjects are shown in Table 1. Compared with controls, more gallbladder cancer cases (72.6\%) and gallstone cases (66.7\%) were female.

Gallbladder cancer, gallstone and bile duct stone cases had higher body mass index (BMI) than controls. All three types of biliary tract cancer had significantly higher proportion of gallstones $(59.6 \%$ - 84.8\%) compared with controls (24.7\%).

Among population controls, the genotype distributions of these 6 SNPs were in HardyWeinberg equilibrium. The risk estimates for biliary tract cancers and biliary stones associated with these DNA repair SNPs are shown in Table 2. Of these 6 SNPs, only the $M G M T$ EX5-25C >T marker was associated with biliary tract cancers. Specifically, carriers of the $\mathrm{CT}$ genotype had a significantly reduced risk of gallbladder cancer $(\mathrm{OR}=0.62 ; 95 \%$ CI 0.40-0.94) compared with those with the $\mathrm{CC}$ genotype. This marker was also associated with a lower risk of bile duct cancer (OR: 0.61; 95\% CI: 0.35-1.06) and ampulla of Vater cancer ( $\mathrm{OR}=0.85 ; 95 \% \mathrm{CI} 0.39-1.87$ ), but the associations were not statistically significant. The associations between the MGMT EX5-25C $>\mathrm{T}$ marker and biliary tract cancers changed little after further adjustment for gallstones. MGMT EX5-25C > T was not associated with biliary stones, nor where any of the other markers examined associated with biliary tract cancers or stones.

We examined the effect of the MGMT EX5-25C>T variant by subjects' smoking behavior, body mass index, gallstone status, and consumption of preserved food, red meat, vegetables, and fruits. No significant interaction by these lifestyle factors was found $(P$ interaction range: 0.1-0.9). In addition, we genotyped $L I G 3$ EX18-75G $>$ A, MGMT EX7+13A>G, MGMT $E X 7+119 A>G$, and $R A D 23 B$ IVS5-57T $>\mathrm{A}$, which we found to be non-polymorphic or had a minor allele frequency less than $1 \%$ in this Asian population.

\section{DISCUSSION}

In this population-based study, we found that the MGMT EX5-25C > T marker in the DNA repair pathway was associated with a reduced risk of biliary tract cancer. No significant associations with biliary tract cancers were observed for the other 5 SNPs examined, although small-to-modest effects of the markers cannot be ruled out because of limited 
sample sizes. Theses results, although in need of confirmation, suggest that variants in $M G M T$ may play a role in biliary tract cancer etiology, particularly for gallbladder cancer.

The MGMT EX5-25C $>\mathrm{T}$ has been shown to be associated with a reduced risk of oral cancer (10) and an increased risk of prostate cancer (11), lung cancer (12), and bladder cancer (13); no association, however, was found for gastric cancer (14) and colorectal polyps (15).

Reasons for the differential effect of this marker in various cancers are unclear but may be related in part to substrate (exposure) specificity for the different organ sites and differences in the frequencies of the variant allele in the study populations.

Our observation that the MGMT EX5-25C>T marker is associated with gallbladder cancer risk is biologically plausible. The protein encoded by the $M G M T$ gene, $\mathrm{O}^{6}$-methylguanineDNA methyltransferase (also named $\mathrm{O}^{6}$-alkylguanine-DNA alkyltransferase), repairs DNA damage such as $\mathrm{O}^{6}$-alkylguanine DNA adducts $(16,17)$ caused by alkylating agents including N-nitroso compounds. These DNA adducts, if un-repaired, cause initiation of mutations and cellular cytotoxic actions (18). In vitro and in vivo data showed that MGMT plays a critical role in protecting cells from mutagenic or carcinogenic action of alkylating agents $(16,17)$. Recent data showed that the MGMT EX5-25C $>\mathrm{T}$ variant was associated with increased mutant frequency in lymphocytes of individuals exposed to alkylating agents (i.e. smokers) (19), suggesting that this evolutionarily conserved, nonsynonymous SNP may alter the phenotype of the MGMT protein resulting in suboptimal repair of $\mathrm{O}^{6}$-methylguanine lesions after exposure to alkylating agents. We, however, did not find any significant modification of the SNP effect by consumption of alkylating agents, including smoking and preserved food.

Strengths and limitations of the study should be noted. Selection bias is minimal due to the high case ascertainment (over 95\%) and high response rate (over 85\%). Misclassification of cancer and stone cases was minimal due to the detailed review of pathology and clinical data; also misclassification of genotypes was minimal, judging from the high concordance and high reproducibility of genotyping results among quality control samples. Despite being the largest biliary tract cancer study to date, the study had limited statistical power to evaluate some main effects, especially for ampulla of Vater cancer, due to the rarity of some high-risk alleles. Because of this, we cannot rule out the possibility of small-to-modest effects of these markers. Since gene coverage was limited and only 6 SNPs were evaluated, the effect of potentially important markers in the DNA repair pathway may have been missed. Also, the observed effect of the MGMT EX5-25C >T marker on biliary tract cancer may represent the effect of other functional SNPs that are in linkage disequilibrium with the MGMT EX5-25C>T locus. Finally, because the population in Shanghai is ethnically homogeneous, our results may not be generalizable to other populations.

In summary, this population-based study in Shanghai showed that the MGMT EX5-25C >T marker was associated with decreased risks of biliary tract cancers, particularly gallbladder cancer. Future studies with larger sample size and broader coverage of the MGMT gene and other genes in the DNA repair pathway are needed to confirm these results and to identify causal gene variants involved in biliary tract cancer etiology.

\section{Acknowledgments}

We thank the collaborating surgeons and pathologists in Shanghai for assistance in patient recruitment and pathology review; Chia-Rong Cheng, Lu Sun, and Kai Wu of the Shanghai Cancer Institute for coordinating data and specimen collection; and Shelley Niwa of Westat for support with study and data management. This research was supported by the Intramural Research Program of the National Institutes of Health and the National Cancer Institute. 


\section{The abbreviations used are}

$\begin{array}{ll}\text { XRCC } & \text { X-ray repair cross complementary group } \\ \text { MGMT } & \mathrm{O}^{6} \text {-methylguanine-DNA-methyltransferase } \\ \text { ERCC } & \text { excision repair cross complementary group } \\ \text { OR } & \text { odds ratio } \\ \text { CI } & \text { confidence interval }\end{array}$

\section{REFERENCES}

1. Hsing AW, Gao YT, Devesa SS, Jin F, Fraumeni JF Jr. Rising incidence of biliary tract cancers in Shanghai, China. Int J Cancer 1998;75:368-70. [PubMed: 9455795]

2. Berwick M, Vineis P. Markers of DNA repair and susceptibility to cancer in humans: an epidemiologic review. J Natl Cancer Inst 2000;92:874-97. [PubMed: 10841823]

3. Wood RD, Mitchell M, Sgouros J, Lindahl T. Human DNA repair genes. Science 2001;291:1284-9. [PubMed: 11181991]

4. Poirier, MC.; Weston, A. DNA Damage, DNA Repair, and Mutagenesis. In: Bertino, JR., editor. Encyclopedia of Cancer. Academic Press; Boston: 2002. p. 79-87.

5. Hsing AW, Bai Y, Andreotti G, Rashid A, Deng J, Chen J, Han TQ, Wang BS, Zhang BH, Shen MC, Fraumeni JF Jr, Gao YT. Family history of gallstones and the risk of biliary tract cancer and gallstones: a population-based study. Int J Cancer 2007;121:832-8. [PubMed: 17450525]

6. Hsing AW, Gao YT, McGlynn KA, Niwa S, Zhang M, Han TQ, Wang BS, Chen J, Sakoda LC, Shen MC, Zhang BH, Deng J, Rashid A. Biliary tract cancer and stones in relation to chronic liver conditions: a population-based study in Shanghai, China. Int J Cancer 2007;120:1981-5. [PubMed: 17278101]

7. Hsing AW, Rashid A, Sakoda LC, Deng J, Han TQ, Wang BS, Shen MC, Fraumeni JF Jr, Gao YT. Gallstones and the risk of biliary tract cancer: a population-based study. Br J Cancer. In Press.

8. Sakoda LC, Gao YT, Chen BE, Chen J, Rosenberg P, Rashid A, Deng J, Shen MC, Wang BS, Han TQ, Zhang BH, Cohen-Webb H, Yeager M, Welch R, Chanock S, Fraumeni JF Jr, Hsing AW. Prostaglandin-endoperoxide synthase 2 (PTGS2) gene polymorphisms and risk of biliary tract cancer and gallstones: a population-based study in Shanghai, China. Carcinogenesis 2006;27:12516. [PubMed: 16361272]

9. Packer BR, Yeager M, Burdett L, et al. SNP500Cancer: a public resource for sequence validation, assay development, and frequency analysis for genetic variation in candidate genes. Nucleic Acids Res 2006;34:D617-21. Database issue. [PubMed: 16381944]

10. Huang WY, Olshan AF, Schwartz SM, Berndt SI, Chen C, Llaca V, Chanock SJ, Fraumeni JF Jr, Hayes RB. Selected genetic polymorphisms in MGMT, XRCC1, XPD, and XRCC3 and risk of head and neck cancer: a pooled analysis. Cancer Epidemiol Biomarkers Prev 2005;14:1747-53. [PubMed: 16030112]

11. Ritchey JD, Huang WY, Chokkalingam AP, Gao YT, Deng J, Levine P, Stanczyk FZ, Hsing AW. Genetic variants of DNA repair genes and prostate cancer: a population-based study. Cancer Epidemiol Biomarkers Prev 2005;14:1703-9. [PubMed: 16030105]

12. Wang L, Liu H, Zhang Z, Spitz MR, Wei Q. Association of genetic variants of O6-methylguanineDNA methyltransferase with risk of lung cancer in non-Hispanic Whites. Cancer Epidemiol Biomarkers Prev 2006;15:2364-9. [PubMed: 17164358]

13. Li C, Liu J, Li A, Qian L, Wang X, Wei Q, Zhou J, Zhang Z. Exon 3 polymorphisms and haplotypes of O6-methylguanine-DNA methyltransferase and risk of bladder cancer in southern China: a case-control analysis. Cancer Lett 2005;227:49-57. [PubMed: 15885889]

14. Huang WY, Chow WH, Rothman N, Lissowska J, Llaca V, Yeager M, Zatonski W, Hayes RB. Selected DNA repair polymorphisms and gastric cancer in Poland. Carcinogenesis 2005;26:13549. [PubMed: 15802298] 
15. Bigler J, Ulrich CM, Kawashima T, Whitton J, Potter JD. DNA repair polymorphisms and risk of colorectal adenomatous or hyperplastic polyps. Cancer Epidemiol Biomarkers Prev 2005;14:25018. [PubMed: 16284370]

16. Deng C, Xie D, Capasso H, Zhao Y, Wang LD, Hong JY. Genetic polymorphism of human O6alkylguanine-DNA alkyltransferase: identification of a missense variation in the active site region. Pharmacogenetics 1999;9:81-7. [PubMed: 10208646]

17. Inoue R, Abe M, Nakabeppu Y, Sekiguchi M, Mori T, Suzuki T. Characterization of human polymorphic DNA repair methyltransferase. Pharmacogenetics 2000;10:59-66. [PubMed: 10739173]

18. Margison GP, Povey AC, Kaina B, Santibanez Koref MF. Variability and regulation of O6alkylguanine-DNA alkyltransferase. Carcinogenesis 2003;24:625-35. [PubMed: 12727789]

19. Hill CE, Wickliffe JK, Guerin AT, Kinslow CJ, Wolfe KJ, Ammenheuser MM, Abdel-Rahman SZ. The L84F polymorphism in the O6-Methylguanine-DNA-Methyltransferase (MGMT) gene is associated with increased hypoxanthine phosphoribosyltransferase (HPRT) mutant frequency in lymphocytes of tobacco smokers. Pharmacogenet Genomics 2007;17:743-53. [PubMed: 17700363] 


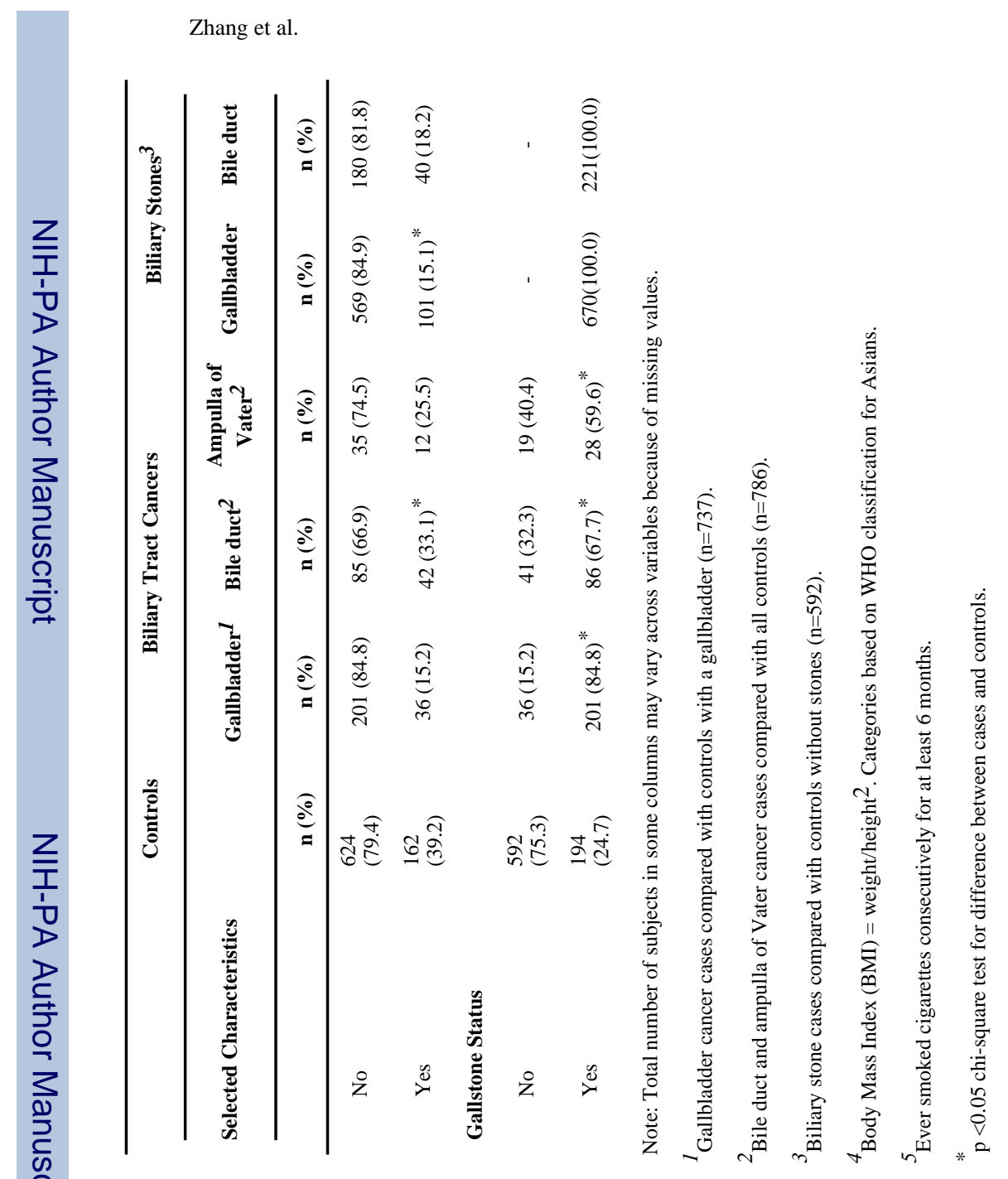




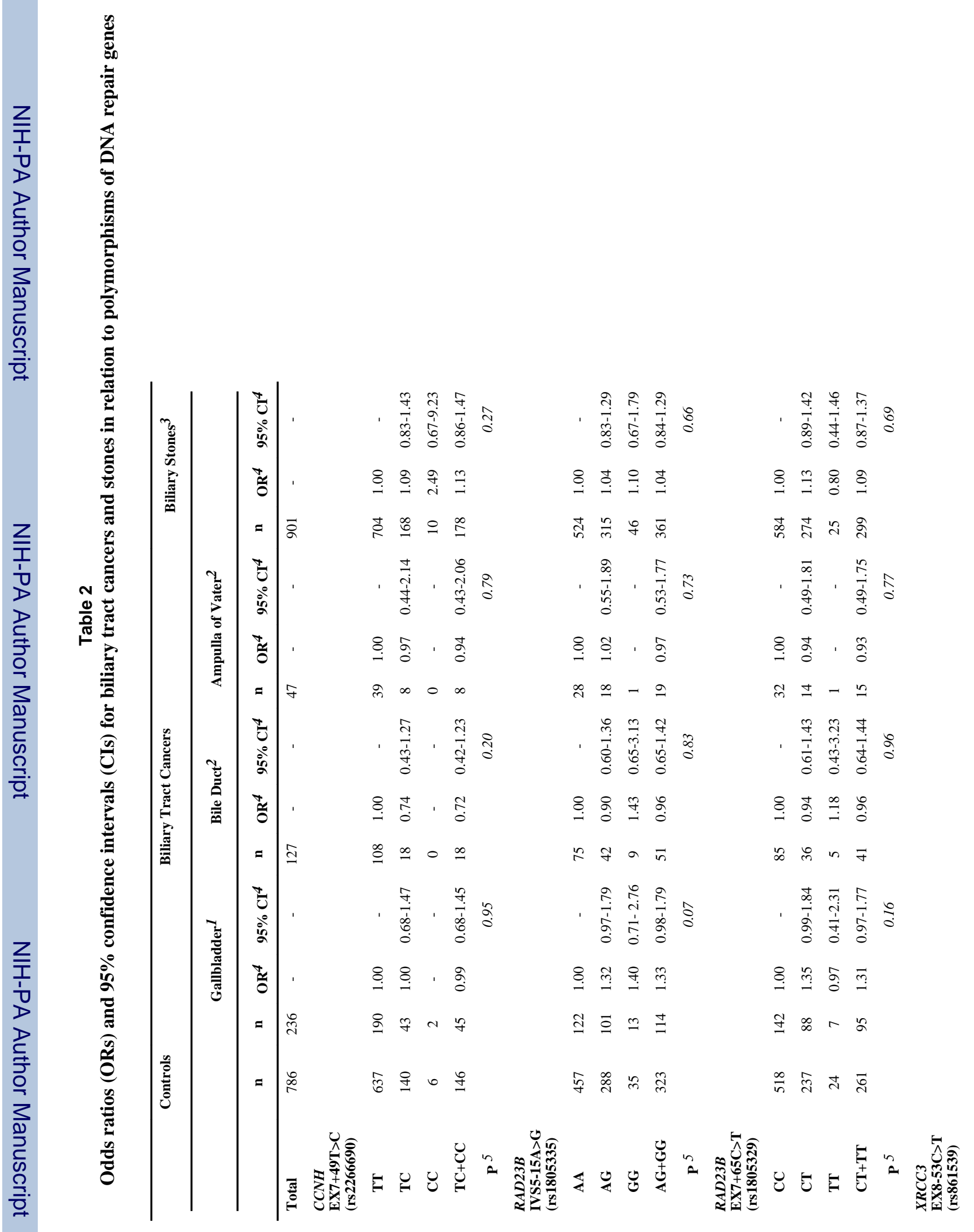




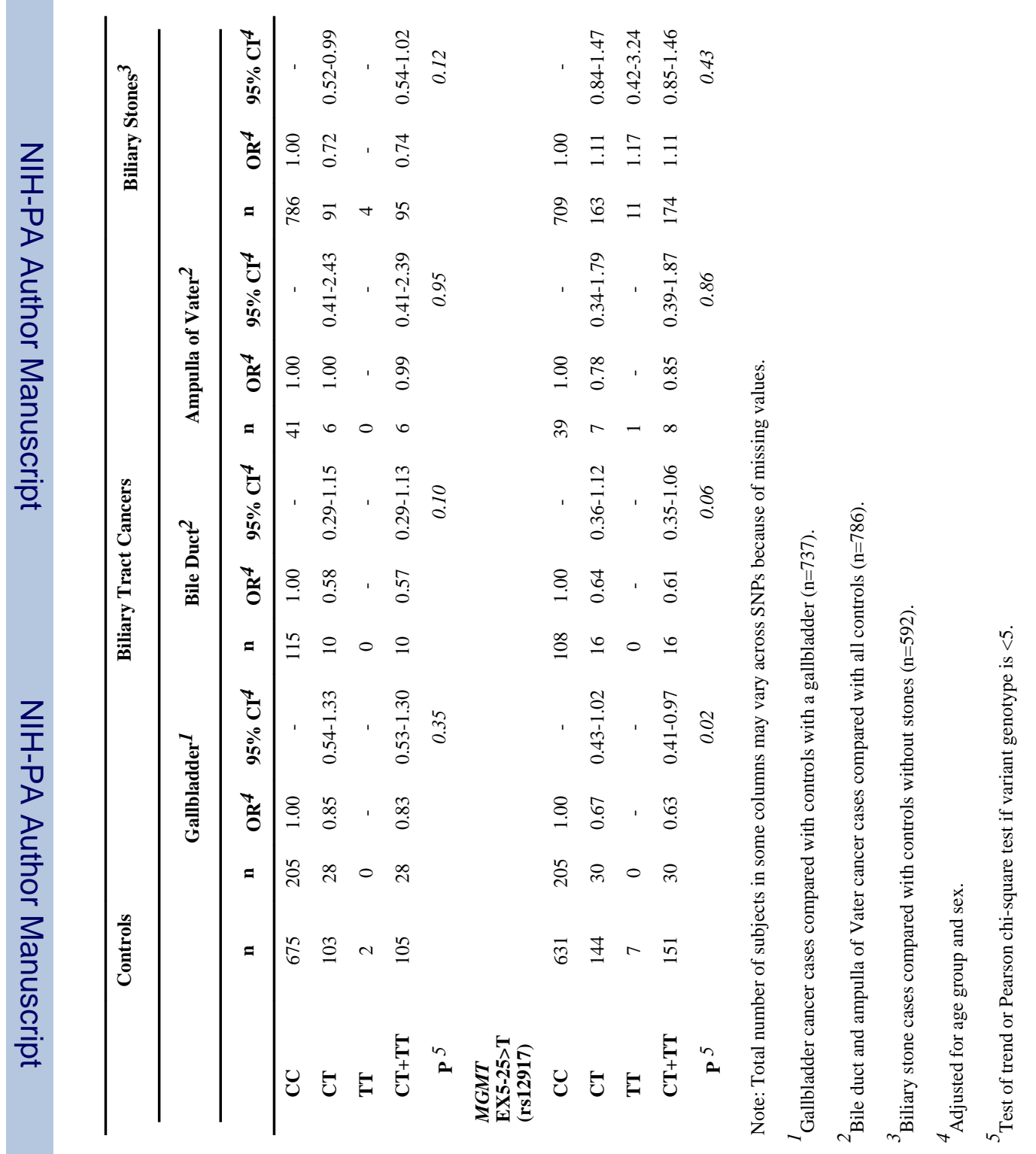

